\title{
SUPPORTER LE TRAVAIL DE LA RELATION?
}

Des professionnels de psychiatrie en grève, des intervenants sociaux de centre d'hébergement pour demandeurs d'asile qui tirent la sonnette d'alarme, des services d'urgence saturés... Les exemples pourraient se multiplier. Des politiques qui apparaissent absurdes aux professionnels, des suppressions de postes, un suicide, peuvent être les déclencheurs d'un " ras-le-bol ». Pour beaucoup d'intervenants, les évolutions organisationnelles ne sont pas sans conséquences et ont des incidences sur les prises en charge et sur les personnes destinataires de l'intervention. Est-ce que les métiers relationnels ont une spécificité ? Doivent-ils obéir aux mêmes logiques que les métiers dits "productifs "? Quelles sont les espaces d'adversité ou de solidarisation possible ? Le travail est-il devenu si insupportable qu'on se pose la question de comment le supporter?

1 Intitulée « La clinique psychosociale et les dispositifs de soutien aux professionnels ». Cette recherche a été financée dans le cadre d'un partenariat entre I'Université Lyon II et le Centre Hospitalier Le Vinatier. Elle a été menée en collaboration entre le Centre de Recherches en Psychopathologie et Psychologie Clinique de l'Université Lyon II (CRPPC) et I'OrspereSamdarra, sous la responsabilité de Georges Gaillard et grâce au travail de Gaïa Barbieri. En plus de ces deux chercheurs, le séminaire a réuni : Khedidja Benarab, Thierry Bruyère, Nicolas Chambon, Stéphane Pawlof, Julien Payet, Adrien Pichon et Bertrand Ravon.

\section{Supporter le travail ?}

Cette question, autant théorique, que pratique nous a animé à l'Orspere-Samdarra dans le cadre d'une recherche et d'un séminaire'. Théorique car la clinique psychosociale qui était au fondement de l'Orspere proposait de partir du malaise des intervenants de première ligne pour tenter d'en faire l'objet d'un travail spécifique. En somme, le trouble ressenti dans l'exercice professionnel constituait l'indice d'une exigence de travail psychique, imposée par la situation de relation intersubjective (Kaës, 2010). Cette exigence, déjà identifiée dans la dynamique transféro-contretransférentielle classique, étant en quelque sorte " chauffée à blanc " et poussée à ses limites, dans le cadre des cliniques de la précarité et du syndrome d'autoexclusion (Furtos, 2009). Inscrire ce malaise professionnel dans un tel cadre de lecture pouvait susciter quelques critiques et ouvrir un débat sur l'objectivité ou la subjectivité du trouble, avec en arrière-plan l'ouverture vers le champ de la responsabilité politique. Une telle dialectique ne tient plus dès lors que l'on considère le plan de la relation intersubjective, ou du champ complexe d'une écologie des relations, comme un objet autant clinique que politique, comme le montre d'ailleurs les articles de cliniciens dans ce numéro.

Mais c'est aussi un questionnement pratique. Une grande majorité de structures font aujourd'hui appel à des superviseurs et autres intervenants en analyse de la pratique professionnelle pour soutenir et accompagner ce travail psychique spécifique : le "soutien aux professionnels " devient un marché à part entière. Et même des chercheurs, des observatoires tels que le nôtre, sont sollicités pour proposer une réponse à la détresse des professionnels et bénévoles. Comment répondre à ces demandes, sachant que la réponse se doit souvent d'être " efficiente " selon les termes de la commande ? Qui l'évalue ? N'y a-t-il pas là un risque de dépoli- 
tisation? Nous développons ainsi formations, séminaires cliniques, analyses de la pratique, échanges autour des pratiques, coordinations... en faisant le constat que cela répond bel et bien à une demande mais sans savoir forcément dans quel cadre "théorique " ce type d'actions s'inscrit. Ainsi le trouble déborde-t-il du champ des pratiques vers celui des cadres conceptuels, et appelle peut-être à apprendre à composer avec celui-ci. "Faire avec le trouble " (Harraway, 2016) serait peut-être la nouvelle exigence du travail psychique imposée par la reconfiguration globale du champ des relations intersubjectives (précarisation généralisée, migrations, transformations des institutions...).

\section{Malaise dans l'organisation}

Le constat dans de nombreuses institutions et dispositifs est sombre. Les mouvements sociaux qui ont cours sont là pour nous le rappeler. "Va te faire soigner!", Lise Gaignard questionne, à partir de sa pratique clinique, comment appréhender la souffrance liée au travail. Sa contribution est déroutante car finalement elle nous questionne sur la psychologisation du malaise dans l'organisation et plus généralement sur le sens même du travail aujourd'hui. Son texte illustre peut être la responsabilité du clinicien de refuser l'assignation implicite à traiter le malaise psychosocial dans une perspective gestionnaire. Et c'est aussi le propos de Frédéric Mougeot pour qui la "nouvelle gestion publique ", la " pression des lits " et l'" attention au flux " ne sont pas sans conséquences pour les intervenants en santé. L'hôpital psychiatrique se concentre aujourd'hui sur les malades en "crise ". En filigrane, on comprend que certaines cliniques (et certains publics ?) ne sont pas adaptées à ce type de contrainte managériale : "L'urgence à faire sortir les patients de l'hôpital dévie les pratiques professionnelles héritées de la psychiatrie de secteur. "En conséquence, et en inadéquation avec ce qui est porté par l'idéal républicain de l'hôpital public, ce sociologue constate une inégalité du traitement : "le soin n'est donc pas déployé de façon égalitaire pour chaque patient. La dimension clinique du travail des soignants n'est mobilisée que dans quelques cas. » On ne peut que partager cette interprétation : l'effort que demandent certains publics peut induire des refus de prise en charge. "La clinique, cœur du métier des soignants de la psychiatrie, jusqu'alors protégée, est aujourd'hui traversée par des contraintes extérieures à la relation de soin. " II importe aujourd'hui d'en prendre acte : la clinique ne peut s'abstraire du contexte social inhérent à son exercice et être totalement " pure ", contrevenant au désir d'indépendance et d'autonomie d'intervenants dans les métiers relationnels (Doucet et Viviers, 2016).

Et c'est le même constat qui mobilise Laura Holdner, qui contribue en tant que " jeune clinicienne ". Le modèle de clinique est mis à l'épreuve de certaines contraintes notamment liées à l'organisation même et ce qu'elle implique en termes de temporalité. L'expression du malaise est-elle ici à entendre comme l'indice de cette nécessité de "faire avec le trouble " ? Mais comment dès lors en faire l'objet de l'élaboration d'un positionnement professionnel spécifique, sans pour autant abdiquer dans une posture sacrificielle ou masochique ? Faudrait-il " apprendre à encaisser " et " se faire le cuir " pour construire son positionnement clinique dans 
le contexte actuel ? L'enjeu n'est-il pas de subvertir cette perspective de survie du plus apte dans un contexte de compétition généralisée, pour faire émerger de nouvelles formes de collaboration, de nouvelles manières de faire collectivement avec le trouble ? D'un indice de stress dans un paradigme de compétition, le trouble deviendrait alors celui d'une recomposition profonde, sur les plans conceptuels et pratiques, des cadres d'exercice professionnel.

Bien qu'il y ait un rejet partagé des visées managériales, nous ne pouvons pas réduire les problématiques actuelles qui traversent les métiers relationnels à un problème d'organisation. Si l'écart entre les " prescriptions " de l'encadrement et le travail réel des professionnels est maintenant bien documenté, il y a lieu de souligner que cet écart empêche. Mais comment faire face ? Nous avons identifié deux dimensions dans l'embarras sur les situations de travail, mais qui sont autant de prises pour le supporter.

\section{En quête de sens}

L'évolution des politiques, la désinstitutionalisation, le "virage ambulatoire " en psychiatrie, les politiques par " appel d'offre " bouleversent les missions des intervenants en santé et sociaux. Mais accompagner et soigner des personnes implique aussi l'engagement de l'intervenant dans la relation. II est par exemple attendu des " infirmières " qu'elles associent technicité et empathie, cure et care, qu'elles s'attachent pour soigner (Molinier, 2009) et des intervenants sociaux qu'ils considèrent des personnes dans toute leur singularité, au plus près de leur problème, sans avoir un a priori normatif sur ce qu'il convient de faire.

Mais quel est alors le " mandat " ? Qui l'édicte ? Le " sens " du travail n'irait plus forcément de soi ; " auto-conceptualisé en situation " (Soulet, 2016). Cela est très bien présenté et synthétisé par Bertrand Ravon et Pierre Vidal-Naquet qui prennent l'exemple du travail social. Ils définissent alors la professionnalité comme une " capacité issue de l'expérience, qui permet à des professionnels, à la fois de respecter les règles du métier et de les transgresser afin de s'adapter aux situations ". Mais qui aujourd'hui est à même de "valider " ces transgressions ? Le cadre ? Dans un monde où la disruption est devenue une valeur cardinale, la transgression ne tend-elle pas à faire règle ? On aurait peut-être trop vite fait de prendre cela comme l'expression ultime d'une perversion. Nous rencontrons à l'observatoire des intervenants travaillant dans des dispositifs de " mise à l'abri " sans fiche de poste et qui ne savent pas vraiment ce qui est attendu d'eux par leur hiérarchie, si ce n'est de parer au plus urgent.

II importe alors de prendre en considération l'objet même du travail relationnel, d'en faire le lieu d'une élaboration; une recherche en tant que telle. Comment soigner à l'Unité pour Malade Difficile (UMD) ? Voici la question que nous partage Slimane Zerrouk à partir d'une étude menée dans le service : qu'est-ce qui rend ce dispositif thérapeutique ? Surtout, comment donner du sens, pour des infirmiers, à une pratique d'ordre clinique en contexte sécuritaire ? Les réponses semblent justement résider dans la capacité d'un collectif, d'une équipe, à traverser le trouble, 
la crise permanente et à produire une nouvelle générativité pratique. L'appui d'un collectif vivant, créatif, serait alors la condition pour transformer les " faits divers en faits cliniques "(Grondin, 2015). La recherche de sens serait alors un commun, une question ouverte sur le collectif, traversée par la conflictualité comme on disait classiquement. On pourrait maintenant proposer qu'elle soit une manière de produire des formes hybrides, temporaires, à même d'orienter une pratique incertaine, sur un mode rhizomatique : un devenir équipe en somme, tendu vers un horizon toujours fuyant. On comprend aussi qu'une telle éthique soulève sa part d'inconfort dans ce contexte précis : "L'incertitude et la surprise font partie du quotidien des soignants » nous dit-il.

S'agit-il alors de faire reconnaître ce travail " invisible " selon le souhait de JeanPaul Lanquetin ? Pour cet infirmier, prendre soin c'est aussi " produire " des attentions et des liens. Pour Hélène Liotard, l'incertitude est plutôt lue comme un principe du travail interinstitutionnel. Celui-ci, écrit-elle, " ouvre donc un "jeu de visibilité-invisibilité" entre les professionnels et leurs institutions de référence ". II faut dire qu'il ne faut pas avoir trop de certitude quand on travaille dans le champ de la santé mentale et que c'est un travail que de remodeler ses frontières! La santé mentale concerne de nombreux acteurs et pas seulement dans le champ médical. II importe alors d'œuvrer au travail partenarial pour prendre au mieux en charge le public : "Pour les professionnels de terrain, la question du décloisonnement fait plus écho à un souci pratique du quotidien pour mettre en œuvre leur mission qu'à un défi politique à mener. Ils ont besoin de connaître les autres acteurs de leur territoire et de développer des réseaux de partenaires dans le souci d'orienter au mieux les publics qu'ils accompagnent. »

Céline Gabarro interroge, quant à elle, le travail des agents de guichet de l'assurance maladie et le sens qu'ils donnent à leur mission. Et il est intéressant de lire que la " présence du public permet aussi aux guichetiers de donner un intérêt à leur travail : pouvoir s'impliquer dans des dossiers, chercher une solution de manière visible et donc exercer un rôle plus social et être reconnu par l'usager pour ce zèle et cet investissement personnel en leur faveur ». Des agents dérogent ainsi par moment aux règles en vue "d'obtenir une certaine reconnaissance des demandeurs pour qui ils effectuent ainsi un traitement privilégié ". Mais voilà un paradoxe : alors que les agents valorisent leur mission " en rapport avec la figure de l'usager et via le recours au service rendu ", il est difficile pour ces agents de recevoir les personnes en situation de précarité du fait de la complexité de leur dossier.

En contre-point, deux articles dénotent. Dans un cadre contraint, et dans une institution totale qu'est la prison, Fabrice Guilbaud démontre comment pour les prisonniers, c'est le travail qui devient support. Quand bien même on aurait affaire à un travail purement productif, celui-ci devient une ressource sociale. II permet de coopérer et de se sentir utile. Pour ce sociologue, "à travers leurs pratiques collectives dans l'espace de l'atelier et le rapport entretenu avec leur travail, les travailleurs détenus affirment l'irréductibilité de leur individualité, se posent comme sujets et, in fine, défendent leur liberté et leur intégrité. "

À l'opposé, Emilie Perault nous présente une expérimentation - en cours - d'une 
ferme d'accueil, composée d'une recyclerie ; avec pour idéal fondamental un refus du travail et ses liens de subordination, pour rendre effective une " activité-rotation ", sans "frontière rigide entre les aidants et les aidés ". Ce qui est intéressant à noter, c'est qu'en pratique cet idéal est une épreuve à part entière. Le lecteur est invité à la partager et à suivre l'expérimentation, les interrogations qu'elle pose. D'ailleurs, devant certains incidents, il est décidé de "revenir au Code du Travail ". En creux cela nous interroge justement sur la conformité attendue, que l'on soit aidant ou aidé... comme si la visée politique est ici à même de dissoudre les appétences des individus.

\section{À la recherche du collectif soutenant}

Le sens du travail de la relation se loge donc, en grande partie, dans les effets prétendus ou constatés du côté de la personne destinataire de la relation. Mais il ne peut prendre véritablement " sens " que s'il est élaboré, validé collectivement. Mais quel est ce collectif tant désiré ? La question était déjà posée par Christian Laval et Bertrand Ravon dans la revue Rhizome n²5 "Réinventer l'institution ", publiée en 2006. Dix ans après, on peut affirmer que la perte de sens déjà thématisée s'est massifiée.

Prenons acte que l'institution apparaît de moins en moins soutenante pour de nombreux intervenants. Pour sûr, des professionnels sont en recherche d'espaces pour sortir de l'isolement. Devoir prendre en charge des situations de plus en plus complexes, avoir l'impression d'être l'unique responsable du traitement du problème, fragilise a priori «le " collectif. Pour Bertrand Ravon et Pierre Vidal-Naquet " les épreuves politico-éthiques érodent la dimension collective et politique du travail social et renvoient les professionnels à leurs propres éthiques personnelles pour traiter des situations devenues indécidables. " On comprend alors le glissement souhaité pour que ces épreuves deviennent des ressources, et pour qu'on retrouve des espaces collectifs pour les partager.

Pour Myriam Zittoun, psychologue du travail, intervenante en clinique de l'activité, " les conflits de métiers au centre des discussions entre protagonistes [sont] une porte d'entrée prometteuse. " II importe, en ayant en vue une réorganisation pratique (l'intervenant participe à la mise en œuvre d'alternatives organisationnelles) de nourrir la controverse professionnelle sur la qualité du travail, ou encore la controverse sociale sur le " travail bien fait ». La " dispute professionnelle " permet donc de donner des prises à la transformation des situations de travail et au développement du pouvoir d'agir des professionnels et des collectifs de travail. Myriam Zittoun précise alors : "Du coup, la personne n'est plus seulement dans un collectif, c'est le collectif qui glisse dans la personne. II s'y reconvertit en dialogue intérieur au service de son activité propre. Le collectif, dont il est ici question, permet à chacun de "prendre des libertés » avec lui. Ce collectif-là n'est plus seulement une appartenance à honorer ou une contenance à adopter, mais un instrument de travail sur le travail, très personnel, à user et à "maintenir" ensemble. »

Laissons les mots de la fin à Gaïa Barbieri et Georges Gaillard qui, dans une pers- 
pective critique, caractérisent le "marché " de l'analyse de la pratique pour mieux nous inviter à participer à de "nouveaux " espaces d'étayage à même de restaurer de la responsabilité partagée. Cet article illustre bien les impasses qui s'ouvrent à vouloir faire d'un processus vivant et en large part mystérieux (la nécessité pour tout individu d'être dans un processus d'autocréation permanente en lien avec son environnement social, professionnel, non-humain...), une injonction, un slogan productiviste, un mantra automotivationnel. La "fatigue d'être soi " est une réaction contre cette injonction. La dépression, le burn out seraient-ils les ultimes réactions de défense de l'élan vital lorsqu'on tenterait de l'assujettir contre son gré à toute forme d'emprise ? Mais de la même manière, le recours à l'étayage sur le collectif ne se décrète pas... et serait l'objet même d'un travail!

\section{Des absences remarquables}

Concluons donc cette introduction en soulignant deux absences remarquables. La première serait plutôt l'insuffisance de contributions thématisant et problématisant les interventions dans les lieux de la précarité (au sens large) alors qu'à l'observatoire nous faisons le constat de l'augmentation de dispositifs fragilisés par l'évolution des modes de financements et par la difficulté à prendre en charge dans de bonnes conditions le public. Ceci n'était pas un choix éditorial : il témoigne de la difficulté à pouvoir élaborer dans un contexte où tout un chacun parait de plus en plus "sous pression". Des professionnels, des bénévoles (parfois au prix de tensions entre eux) agissent " jusqu'à l'épuisement ". Le continuum de leur mission est souvent très large (entre accompagner a minima ou chercher à répondre à toutes les problématiques constatées). Par exemple, sur de nombreux territoires, l'augmentation quantitative du nombre de mineurs non-accompagnés (MNA) induit une re-composition en urgence des dispositifs de prise en charge, qu'ils soient à l'initiative de la puissance publique, d'associations ou d'organisations humanitaires. Les missions se partagent, questionnant la spécialisation des structures et des professionnels et bénévoles qui interviennent en leur sein. D'ailleurs, ces évolutions sont aussi "qualitatives". II y aurait de plus en plus de personnes avec des troubles de santé mentale (Einhorn-Jardin, 2018) dans les dispositifs d'hébergement et/ ou d'accueil des plus précaires. Cela interroge alors la place des intervenants en santé mentale dans (ou aux côtés) de ces dispositifs et leurs profils : quels professionnels sont les plus à même de répondre à la souffrance psychosociale ou liée à des expériences de vie parfois traumatiques ? C'est d'ailleurs un des enjeux de certains programmes innovants (comme ceux s'inscrivant dans les politiques de "logement d'abord "). Mais s'ils permettent de mobiliser de nombreux professionnels qui peuvent avoir des désillusions quant à leurs pratiques actuelles, et sont aussi à ce titre salutaire, ils peuvent aussi les mettre dans une dynamique de concurrence préjudiciable.

La seconde absence concerne celle de personnes concernées par la précarité et/ ou traversées par des troubles de santé mentale, pour qui savoir comment appréhender et supporter le travail est souvent une question centrale tant dans l'avènement du trouble que dans le parcours de rétablissement. Autrement dit, le travail 
(ou son absence) peut être à l'origine d'une rupture dans un parcours de vie, possiblement déclencheur du trouble, mais aussi le moyen par lequel une autre rupture - inscrite elle dans une autre perspective - se réalise. C'est alors souvent la qualité relationnelle du travail qui est cœur : la relation peut justement dégrader la santé du travailleur, ou alors la soutenir... nécessitant accompagnement et adaptation du travail. Pour des personnes en situation de vulnérabilité, la question n'est pas forcément de supporter le travail mais de se protéger d'un travail qui potentiellement peut affecter psychiquement (Martin, 2017).

Nous faisons ici l'hypothèse que cette absence est en partie en lien avec notre manière de poser la question du support au travail relationnel. Elle sous entendait possiblement un sens assez univoque de la relation : une intervention d'un professionnel à destination d'un usager ou patient. Le changement de paradigme souhaité par de nombreux acteurs qui se réclament du rétablissement peut périmer certaines revendications de professionnels qui feraient l'économie d'intégrer celles des personnes concernées par l'intervention. Au regard de ce constat, nous pouvons entrevoir plusieurs perspectives. La première, qui s'inscrit assez facilement dans la suite de ce numéro², porte sur le développement des activités de médiation et des métiers de médiateurs avec tous les enjeux que cette professionnalisation peut induire particulièrement pour les " médiateurs de santé pair " en santé mentale (Demailly, 2014). La seconde perspective consiste à s'autoriser à penser mettre le travail entre parenthèse. Faut-il-sauver le travail ? Si la question se pose aujourd'hui dans l'espace productif (avec la raréfaction du travail épanouissant et émancipateur), notamment avec le développement de l'intelligence artificielle ; suscitant une forte inquiétude sociale, elle commence à faire débat notamment dans le champ de la santé et du travail social. Et si les politiques d'inclusion étaient si efficientes qu'elles nous permettraient d'envisager que le support de la relation ne soit plus le travail ?

\section{BIBLIOGRAPHIE}

Demailly, L. (2014). Les

médiateurs pairs en santé mentale. La nouvelle revue du travail. Répéré à : http:// journals.openedition.org/ nrt/1952.

Doucet, M.-C. et Viviers, S. (2016). Métiers de la relation. Nouvelles logiques et nouvelles épreuves du travail. Laval : Presses de l'université de Laval.

Einhorn-Jardin, L. (2018).

Précarité, parcours

d'hébergement et santé mentale. Une étude dans les CHRS de l'Ain. Rapport d'étude. Orspere-Samdarra.

2 Les Cahiers de Rhizome qui paraittra en 2019 portera sur« Médiations en santé 》
Haraway, D. J. (2016). Staying with the trouble: making kin in the Chthulucene. Durham : Duke University Press.

Grondin, P. (2015). Destins de la sensorimotricite dans le transfert du transfert : vers une symbolisation institutionnelle. Nouvelles perspectives sur les dispositifs de soin institutionnel dans les autismes et la psychose infantile (Thèse en psychologie, sous la direction de R. Rousillon, soutenue le 8 décembre 2015 à l'Université Lumière Lyon II).

Kaës, R. (2010). Pulsion et intersubjectivité. L'exigence de travail psychique imposée par la subjectivité de l'objet: La question des pulsions dans le lien intersubjectif. Dans M. Pichon et H. Vermorel, L'expérience du groupe :
Approche de l'œuvre de René Kaës. Paris : Dunod, 217-228.

Martin, A. (2017). Travailler pour s'en sortir ?. Rhizome, 65-66, (3), 13-14.

Molinier, P. (2009). Temps professionnel et temps personnel des travailleuses du care : perméabilité ou clivage ?. Temporalités. Repéré à : https://journals.openedition. org/temporalites/988

Soulet, M.-H. (2016).

Le travail social, une activité d'auto-conception professionnelle en situation d'incertitude. Sociologies. Dossiers, Relation d'aide et de soin et épreuves de professionnalité. Repéré à : http://journals.openedition.org/ sociologies/5553 